\title{
Distinct Properties of Stimulus-Evoked Bursts in the Lateral Geniculate Nucleus
}

\author{
Henry J. Alitto, ${ }^{1}$ Theodore G. Weyand, ${ }^{2}$ and W. Martin Usrey ${ }^{1}$ \\ ${ }^{1}$ Center for Neuroscience, University of California, Davis, California 95616, and ${ }^{2}$ Department of Cell Biology and Anatomy, Louisiana State University \\ Medical Center, New Orleans, Louisiana 70112
}

\begin{abstract}
Neurons in the lateral geniculate nucleus (LGN) of the thalamus produce spikes that can be classified as burst spikes and tonic spikes. Although burst spikes are generally associated with states of sleep and drowsiness, bursts may also play an important role in sensory processing. This study explores the stimulus properties that evoke burst and tonic spikes and examines the reliability of LGN neurons to produce visually driven bursts. Using reverse-correlation techniques, we show that the receptive fields of burst spikes are similar to, but significantly different from, the receptive fields of tonic spikes. Compared with tonic spikes, burst spikes (1) occur with a shorter latency between stimulus and response, (2) have a greater dependence on stimuli with transitions from suppressive to preferred states, and (3) prefer stimuli that provide increased drive to the receptive field center and even greater increased drive to the receptive field surround. These differences are not attributable to the long interspike interval that precedes burst spikes, because tonic spikes with similar preceding interspike intervals also differ from burst spikes in both the spatial and temporal domains. Finally, measures of reliability are significantly greater for burst spikes than for tonic spikes with similar preceding interspike intervals. These results demonstrate that thalamic bursts contribute to sensory processing and can reliably provide the cortex with information that is similar to, but distinct from, that of tonic spikes.
\end{abstract}

Key words: thalamus; vision; visual; bursts; LGN; cat; coding

\section{Introduction}

At the heart of the retinogeniculocortical pathway are neurons in the lateral geniculate nucleus (LGN) of the thalamus. These neurons receive monosynaptic input from retinal ganglion cells and give rise to axons that terminate in primary visual cortex. Because LGN neurons are strongly driven by the retina and have receptive fields much like those of their retinal afferents (Bishop et al., 1958; Hubel and Wiesel, 1961; Cleland et al., 1971; Mastronarde, 1987; Usrey et al., 1999), the LGN is generally regarded as a structure that simply relays retinal activity without doing much in terms of visual processing. Closer examination, however, reveals a more complex picture of LGN function as LGN neurons dynamically filter and transform the temporal structure of their retinal spike input (for review, see Usrey, 2002). In particular, LGN neurons produce spikes that fall into two categories: burst spikes and tonic spikes (Jahnsen and Llinás, 1984a,b; Guido et al., 1992; Lu et al., 1992). Given the range of views surrounding a role for burst spikes in sensory processing (Sherman, 2001; Steriade,

Received Aug. 17, 2004; revised Nov. 15, 2004; accepted Nov. 20, 2004.

This work was supported by National Institutes of Health Grants EY13588 (W.M.U.), EY12576 (Vision Core Grant), and EY15387 (Vision Training Grant), the McKnight Foundation (W.M.U.), the Esther A. and Joseph Klingenstein Fund (W.M.U.), and the Alfred P. Sloan Foundation (W.M.U.). We thank Farran Briggs and Kimberley McAllister for insightful comments on previous versions of this manuscript and Kelly Henning, Austin Collins, and Daniel Sperka for expert technical assistance.

Correspondence should be addressed to W. Martin Usrey, Center for Neuroscience, University of California, Davis, 1544 Newton Court, Davis, CA 95616. E-mail: wmusrey@ucdavis.edu.

D0I:10.1523/JNEUROSCI.3369-04.2005

Copyright $\odot 2005$ Society for Neuroscience $\quad$ 0270-6474/05/250514-10\$15.00/0 2001a), the question arises, what are the visual response properties of burst spikes?

Although thalamic bursts are prevalent during sleep and drowsiness and likely play a role in disconnecting the cortex from its sensory afferents (Steriade and Llinas, 1988; McCormick and Feeser, 1990; Steriade et al., 1990; McCormick and Bal, 1994; Steriade, 2001b), evidence indicates that thalamic bursts may also serve to convey information to the cortex during sensory processing. For instance, results indicate that bursts are not only capable of faithfully encoding information about the periodicity of grating stimuli (Guido et al., 1992; Lu et al., 1992), but are also well suited for stimulus detection (Guido et al., 1995; Guido and Sherman, 1998). Along these lines, linear systems reconstruction techniques reveal that burst spikes are approximately equal to tonic spikes in their ability to estimate a visual stimulus (Reinagel et al., 1999). Although little is known about the spatiotemporal receptive field properties of burst spikes, a recent study reports that burst spikes have receptive-field centers that are smaller in size compared with tonic spikes (Rivadulla et al., 2003).

Here, we compare the spatial and temporal properties of stimuli that evoke burst and tonic spikes and examine the reliability of burst spikes for coding visual information. Our results show that the receptive fields of burst spikes are similar to, but significantly different from, the receptive fields of tonic spikes. In particular, burst spikes display a shorter latency between stimulus and response, have a greater dependence on stimuli with transitions from suppressive to preferred states, and generally follow stimuli with stronger center and surround stimulation. Our results also 
show that burst spikes can be highly reliable. Previous studies examining the efficacy of thalamocortical communication report that bursts are more effective than tonic spikes at driving cortical responses (Swadlow and Gusev, 2001). Results from the present study add to this work and demonstrate that these highly effective spikes can reliably carry distinct visual information to cortex.

\section{Materials and Methods \\ Surgery and preparation}

Twenty adult cats were used in this study. All surgical and experimental procedures conformed to National Institutes of Health guidelines and were performed with the approval of the Animal Care and Use Committee at the University of California, Davis. Surgical anesthesia was induced with ketamine $(10 \mathrm{mg} / \mathrm{kg}$, i.m.) and maintained with thiopental sodium (20 mg/kg, i.v., supplemented as needed). Animals received a tracheotomy and were placed in a stereotaxic apparatus in which temperature, electrocardiogram, EEG, and expired $\mathrm{CO}_{2}$ were monitored continuously throughout the experiment. The level of anesthesia was maintained by a continuous infusion of thiopental sodium $\left(2-3 \mathrm{mg} \cdot \mathrm{kg}^{-1} \cdot \mathrm{hr}^{-1}\right.$, i.v. $)$. If physiological monitoring indicated a low level of anesthesia, additional thiopental was given, and the rate of continuous infusion was increased. The nictitating membranes were retracted with $10 \%$ phenylephrine, and the pupils were dilated with $1 \%$ atropine sulfate. The eyes were glued to posts attached to the stereotaxic frame to minimize eye movements. The eyes were then refracted, fitted with appropriate contact lenses, and focused on a tangent screen located $172 \mathrm{~cm}$ in front of the animal. The positions of area centralis and the optic disk were plotted by backprojecting the retinal vasculature of each eye onto the tangent screen. A midline scalp incision was made, and wound margins were infused with lidocaine. A small craniotomy was made above the LGN, and the dura was removed. Once all surgical procedures were complete, animals were paralyzed with vecuronium bromide $\left(0.2 \mathrm{mg} \cdot \mathrm{kg}^{-1} \cdot \mathrm{hr}^{-1}\right.$, i.v. $)$ and were mechanically respired.

\section{Data acquisition and visual stimuli}

Recordings were made from neurons in layers A and A1 of the LGN using either pargylene-coated tungsten electrodes (AM Systems, Everett, WA) or borosilicate glass-coated tungsten electrodes (home made). Neuronal responses were amplified, filtered, and recorded to a personal computer equipped with a Power 1401 data acquisition interface and the Spike 2 software package (Cambridge Electronic Design, Cambridge, UK). Spike isolation was based on waveform analysis and the presence of a refractory period, as indicated by the autocorrelogram (Usrey et al., 2000, 2003).

Visual stimuli were created with a VSG2/5 visual stimulus generator (Cambridge Research Systems, Rochester, UK) and were presented on a gamma-calibrated Sony (Tokyo, Japan) monitor running at $140 \mathrm{~Hz}$. The mean luminance of the monitor was 38 candelas $/ \mathrm{m}^{2}$.

White-noise stimuli. A binary white-noise stimulus was used to map quantitatively the spatial receptive fields of LGN neurons. The whitenoise stimulus consisted of a $16 \times 16$ grid of black and white squares. Each square was independently modulated in time according to an m-sequence of length $2^{15}-1$ (Reid and Shapley, 1992; Sutter, 1992; Reid et al., 1997). The stimulus was updated either every frame $(7.1 \mathrm{msec})$ or fourth frame of the display $(28.5 \mathrm{msec})$, and the entire sequence $(\sim 4$ or $16 \mathrm{~min}$ ) was typically repeated several times. The size of the individual squares in the stimulus was optimized for each neuron such that individual squares were small enough to map receptive fields with a reasonable level of detail, yet large enough to map the full spatial extent of the receptive field.

Noise-modulated, contrast-reversing, sine-wave stimuli. To quantify the time course of visual responses, LGN neurons were excited with a contrast-reversing sine-wave grating stimulus (100\% contrast, optimal spatial frequency, and size) that was modulated in time by an $\mathrm{m}$-sequence of length $2^{15}-1$. Specifically, the phase of the grating would change by $180^{\circ}$ each time two sequential terms of the m-sequence were different. Although the temporal information obtained from this stimulus could have been calculated from the more traditional white-noise stimulus (described above), the contrast-reversing sine-wave stimulus evoked a higher percentage of burst spikes ( $\sim 10 \mathrm{vs} 2 \%)$ and therefore provided a higher degree of temporal resolution than could be obtained with the $16 \times 16$ grid of squares. The increased number of bursts made available using the reversing sine stimulus was particularly important for the temporal analysis in which the spike-triggered average was examined at 1.5 msec resolution.

Contrast-reversing sine-wave stimuli: repeating stimuli. To investigate the temporal reliability of thalamic burst spikes, we presented several repeats (generally 100 or more) of a 5 sec clip of the m-sequence modulated, contrast-reversing stimulus. Each presentation of the $5 \mathrm{sec}$ clip was followed by a $5 \mathrm{sec}$ interval of mean gray before the clip was repeated.

\section{Statistical analysis}

Statistical tests. When statistical analysis was required to compare two distributions, we first used Lilliefors modification of the KolmogorovSmirnov test to determine whether the distributions in question were significantly different from normal distributions of unspecified mean and variance $(\alpha=0.05)$. If the distributions were not statistically different from normal, then a $t$ test was used to compare the means of the two populations. However, if the populations were statistically different from normal distributions, then a Wilcoxon rank-sum test or a sign test was used in place of a $t$ test.

Identifying burst and tonic spikes. Bursts were identified according to the criteria of Sherman and colleagues (Guido et al., 1992; Lu et al., 1992; Sherman, 1993). Each burst included a single cardinal spike and one or more subsequent burst spikes. Cardinal spikes were defined as all spikes that were preceded by an interspike interval (ISI) $>50 \mathrm{msec}$ and followed with an ISI $<4 \mathrm{msec}$. After the cardinal spike, subsequent burst spikes were defined as all consecutive spikes with preceding ISIs $<4 \mathrm{msec}$. As soon as the ISI became $>4 \mathrm{msec}$, a burst was considered complete. Only the cardinal spike in a burst was used for analysis in this study. To determine whether any differences in the visual properties of burst and tonic spikes resulted from the long ISI that precedes bursts, a final category of spikes, long-ISI tonic spikes, was identified and used for analysis. LongISI tonic spikes were a subset of tonic spikes that met the first criterion of a burst (ISI $>50 \mathrm{msec}$ ) but not the second criterion (ISI $<4 \mathrm{msec}$ ). For each neuron, the long-ISI tonic spikes were selected so that, on average, their preceding ISIs matched those of the burst spikes ( $\pm 4 \mathrm{msec}$ ).

Spatial maps. Receptive field maps of burst and tonic spikes were made from responses to the $16 \times 16$ white-noise stimulus (described above). This was accomplished by first sorting all of the spikes from a given neuron into two categories, burst spikes and tonic spikes (described above), and then separately performing reverse-correlation analysis on spikes from each category (Citron et al., 1981; Jones and Palmer 1987; Reid et al., 1997; Wolfe and Palmer, 1998). Before performing the reverse-correlation analysis, however, we randomly selected a subset of the tonic spikes so that the number of tonic spikes and burst spikes contributing to the generation of a receptive field map was equal. This selection process was necessary to exclude the possibility that any differences measured between burst and tonic receptive fields were attributable to differences in sample size. Receptive field maps were also made for a subset of the tonic spikes, long-ISI tonic spikes (described above), that met the first criterion of a burst (ISI $>50 \mathrm{msec}$ ) but not the second criterion (ISI $<4 \mathrm{msec}$ ).

Reverse-correlation analysis provides a quantitative description of the receptive fields of a neuron in both space and time. For purposes of analysis, a sliding two-frame average (in time) of the spatial receptive fields was calculated, and the latencies to maximal center and maximal surround responses were determined. Because the receptive field surround is slightly delayed in time relative to the center, these values were sometimes offset by one time bin (the time required to update the whitenoise stimulus). The two-frame averages containing the maximal center and maximal surround response were then fit to two-dimensional, difference-of-Gaussians (DOG) functions, as follows: DOG $=G_{\mathrm{c}}-G_{\mathrm{s}}$, where $G_{\mathrm{c}}$ is the center Gaussian, and $G_{\mathrm{s}}$ is the surround Gaussian. For this analysis, each of the individual Gaussian functions is described by the following equation: $G_{\mathrm{ij}}=K \times \exp \left[-\left(x_{\mathrm{i}}-x_{0}\right)^{2} / 2 \times \sigma^{2}\right] \times \exp \left[-\left(y_{\mathrm{i}}-\right.\right.$ $\left.y_{0}\right)^{2} / 2 \times \sigma^{2}$, where $K$ is the amplitude, $x_{0}$ and $y_{0}$ are the coordinates of the center of the receptive field, and $\sigma$ is the SD of the Gaussian distribution. A constrained nonlinear optimization procedure (MATLAB func- 
tion: fmincon; MathWorks, Natick, MA) was used to minimize the squared error [i.e., $\Sigma$ $(\text { Data }- \text { Fit })^{2}$ ] when fitting spatial maps and all subsequent data sets.

Spike threshold model. To predict possible effects of altering spike threshold on reverse correlation maps, we made a linear model of an LGN neuron with spatial parameters taken from the DOG equation (described above) and temporal parameters taken from the following equation: $K(\mathrm{t})=\alpha^{2} \times t \times e^{-\alpha t}-\beta^{2} \times t \times e^{\beta t}$, where $1 / \alpha=8 \mathrm{msec}$ for the receptive field center and $16 \mathrm{msec}$ for the surround and $1 / \beta=32$ msec. These equations and parameter values have been used previously to model LGN spatiotemporal receptive fields (Chance et al., 1998). We convolved the model LGN neuron with the $\mathrm{m}$-sequence stimulus and simulated the firing rate under two conditions. In the first condition, spike threshold was set so that the model neuron had a firing rate within the range of observed rates. In the second condition, spike threshold was increased to a level in which firing rate decreased by $\sim 50 \%$. Reversecorrelation analysis was performed to calculate receptive field maps under both conditions. Receptive field maps were then fit to a DOG equation to compare and quantify any effects of altering spike threshold on the spatial properties of modeled neurons.

Spike-triggered averages. To compare temporal features of visual responses, spike-triggered averages were calculated from responses to a noise-modulated, contrast-reversing, grating stimulus (described above). The procedure was similar to that used to calculate the spatiotemporal receptive field maps. First, burst spikes, tonic spikes, and longISI tonic spikes were sorted according to the criteria described above. Second, tonic spikes and long-ISI tonic spikes were randomly sampled so that the number of events was equal to the burst spike data set. Finally, reverse correlation was performed separately on each spike category. Spike-triggered averages were interpolated with a cubic spline (MATLAB function: spline; MathWorks), and temporal features of the spiketriggered average were quantified (see Fig. $3 A$ ).

Temporal reliability. To quantify the reliability of LGN bursts, we calculated a reliability index from neuronal responses to a repeating, contrast-reversing stimulus (described above). Given the occurrence of a burst in one repeat of the stimulus, the reliability index represents the probability that a burst occurred at the same time in all other repeats of the stimulus. An index value of 1.0 would indicate that bursts always occurred at the same relative time during different repeats of the stimulus; an index value of 0.0 would indicate that bursts never occurred at the same relative time during different repeats of the stimulus. For this analysis, the time window for burst occurrence ranged from \pm 1 to $\pm 20 \mathrm{msec}$.

\section{Results}

\section{Spatial information conveyed by burst spikes}

We recorded neuronal responses from 32 neurons in layers A and A1 of the cat LGN during the presentation of a white-noise stimulus $(16 \times 16$ pixels $)$ and used reverse-correlation analysis to determine the average stimulus that preceded spikes at different time intervals between stimulus and response (see Materials and Methods). To compare the spatial information conveyed by burst and tonic spikes, we calculated receptive field maps for each neuron using three different subsets of spikes taken from the original spike train of the neuron (Fig. 1) (see Materials and Methods). The first subset of spikes, burst spikes, included only the cardinal spikes of burst responses. The criteria used to identify cardinal burst spikes were (1) a preceding ISI $>50 \mathrm{msec}$ and (2) a subsequent ISI $<4$ msec (Guido et al., 1992; Lu et al., 1992). The second subset of spikes, tonic spikes, was a randomly selected group of tonic spikes that, for purposes of analysis (described below), was equal in number to burst spikes. Finally, the third subset of spikes included only those tonic spikes that met the first criterion of a burst (preceding ISI $>50 \mathrm{msec}$ ) but not the second. This last subset of spikes, referred to as long-ISI tonic spikes, was also matched in number to the number of burst spikes produced by the neuron. A quick inspection of the different receptive field maps shown in Figure 1 reveals that burst and tonic receptive fields are similar to each other in terms of spatial location and center/surround signature (on vs off). Across our sample of 32 neurons, two-dimensional DOG fits revealed an average displacement of $0.07 \pm 0.01^{\circ}$ between the center point of burst and tonic receptive fields. In no case did we see a reversal in center or surround sign (i.e., on vs off).

Although the general features of burst and tonic receptive fields are similar, closer examination shows that the receptive field surround is relatively stronger than the center for burst spikes compared with tonic spikes. To quantify this relationship, we fit the spatial receptive fields of burst and tonic spikes (at latencies between stimulus and response that correspond to maximal center and surround response; see Materials and Methods) to two-dimensional DOG equations and compared the area under each Gaussian. As shown in Figure $2 A$, the average surround to center ratio for burst spikes is greater than the same measure for tonic spikes $(0.27 \pm 0.02$ vs $0.23 \pm 0.01$, respectively; $p<$ $0.05)$. To determine whether the larger surround/center ratio for burst spikes is caused by an increase in surround strength, decrease in center strength, or a combination of both, we compared differences in surround strength of burst and tonic spikes to differences in center strength of burst and tonic spikes. For this 
A

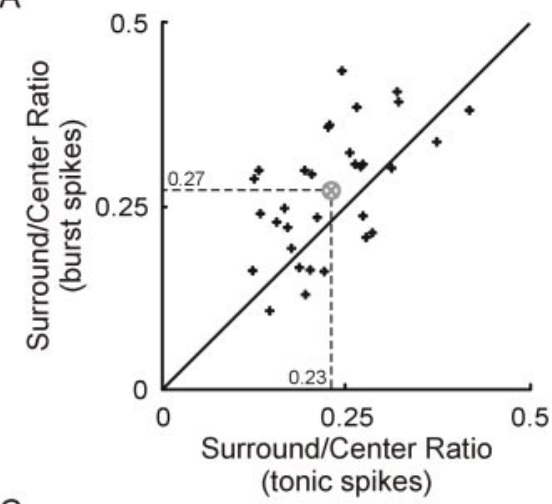

C

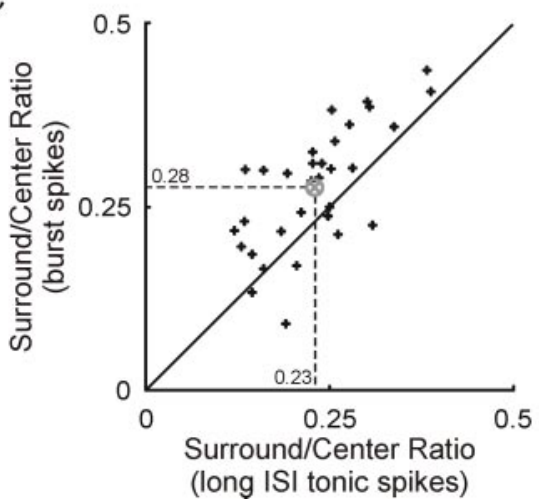

B

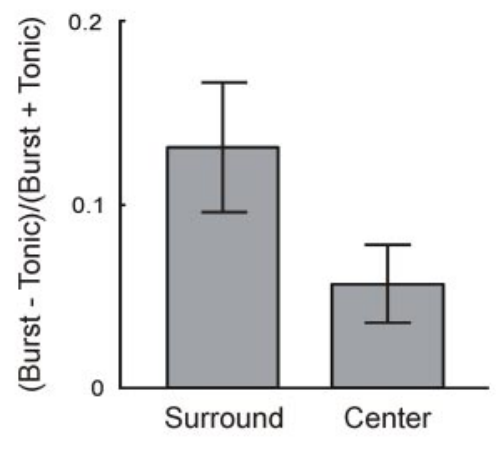

D

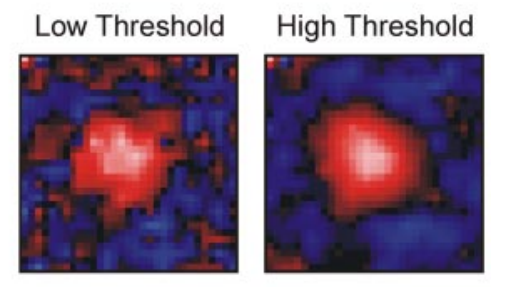

Excited by Dark

Excited by Light

Figure 2. Spatial properties of the average stimulus to evoke burst spikes are significantly different from those that evoke tonic and long-ISI tonic spikes. $A$, The surround/center ratio of the average stimulus to evoke burst spikes is significantly greater than that for tonic spikes. B, Both the surround and center subunits are stronger for burst spikes than for tonic spikes; however, the increase in surround strength is greater than the increase in center strength. Error bars represent SEM. C, The surround/center ratio of the average stimulus to evoke burst spikes is significantly greater than that for long-ISI tonic spikes. D, Spatial maps of a model LGN receptive field convolved with the white-noise stimulus under low spike-threshold and high spike-threshold conditions (see Materials and Methods). The surround/center ratio is greater for the high threshold condition (0.25) compared with the low threshold condition (0.06).

analysis, it was essential that comparisons be made between spike-count matched data sets (see Materials and Methods). The results show that although the strength of both surround and center regions of burst spike receptive fields are greater, on average, than corresponding tonic spike values (Fig. $2 B$ ) (surround $\Delta_{\text {(burst vs tonic) }}=0.129 \pm 0.034, p<0.0001$; center $\Delta_{\text {(burst vs tonic) }}=$ $0.055 \pm 0.020, p<0.01)$, there was a near-significant trend indicating that the increase in surround strength was greater than the increase in center strength $(p=0.065)$.

To determine whether the increased strength of the receptive field surround and center (i.e., area under each Gaussian) of burst spikes is attributable to an increase in the spatial extent of the receptive field subunit and/or an increase in the amplitude of the subunit, we compared space constants $(\sigma)$ and amplitudes of the best-fitting DOG equations (see Materials and Methods). Across our sample of LGN neurons, results show that the increased surround strength of burst receptive fields is caused by an increase in the amplitude of the surround subunit ( $\Delta$ surround amplitude $_{\text {(burst vs tonic) }}=0.155 \pm 0.044 ; p=0.01$ ) and not an increase in the spatial extent of the surround ( $\Delta$ surround $\sigma_{\text {(burst vs tonic) }}=$ $-0.025 \pm 0.037 ; p=0.49)$. Similarly, the increased center strength of burst receptive fields is caused by an increase in the amplitude of the center subunit $(\Delta$ center amplitude (burst vs tonic) $=0.053 \pm 0.016$; $p=0.01)$ and not an increase in the spatial extent of the center $(\Delta$ center $\sigma_{\text {(burst vs tonic) }}=0.013 \pm 0.014 ; p=0.36$ ).

Because one of our criteria for the identification of a thalamic

burst is a long ISI ( $>50 \mathrm{msec})$ preceding the cardinal spike of a burst (Guido et al., 1992; Lu et al., 1992), it is possible that the differences in surround and center strength are a result of this ISI and not the result of any property specific to burst spikes. To investigate this possibility, we compared the receptive fields of burst spikes to the receptive fields of long-ISI tonic spikes (described above). Across our sample of LGN neurons (Fig. 2C), there is a significant increase in the surround to center ratio of burst receptive fields compared with long-ISI tonic receptive fields $(0.28 \pm 0.02$ vs $0.23 \pm 0.01$, respectively; $p<0.05)$. This comparison indicates that differences in the spatial properties of burst and tonic receptive fields are not solely a consequence of the long ISI that precedes bursts.

Although the mechanism that underlies the increased surround to center ratio of the average stimulus to evoke burst spikes is unclear, one likely possibility is a change in spike threshold. To test this possibility, we performed a simple convolution of a model LGN receptive field (see Materials and Methods) with the m-sequence stimulus. This convolution was performed twice, once with a relatively low spike threshold and once with a higher threshold. As shown in Figure 2D, the surround to center ratio for this model neuron was greater for the high-threshold condition relative to the low-threshold condition. This finding is consistent with our result that visually driven burst spikes require more excitation, on average, than visually driven tonic spikes.

\section{Temporal information conveyed by burst spikes}

To compare temporal information conveyed by burst and tonic spikes, we recorded neuronal responses from 35 LGN neurons during the presentation of an $\mathrm{m}$-sequence-modulated, contrastreversing, sine-wave grating and calculated spike-triggered averages using reverse-correlation techniques (see Materials and Methods). The spike-triggered average shows the time course of the average stimulus to precede a neuronal response and generally follows a sequence beginning with a nonpreferred/suppressive stimulus followed by a preferred/excitatory stimulus (Fig. $3 A$ ). For each neuron in our sample, separate spike-triggered averages were made for tonic spikes, burst spikes, and long-ISI tonic spikes. As indicated in the representative examples shown in Figure 3, $B$ and $C$, spike-triggered averages from these categories of spikes differed from each other in several respects.

The excitatory phase of the spike-triggered average for burst spikes was significantly different from that of tonic spikes, both in terms of time course and strength. On average, the latency between excitatory phase maximum and neuronal response was significantly shorter for burst spikes than for tonic spikes (Fig. $\left.4 A_{1}\right)$ (burst spikes $=29.6 \pm 1.0 \mathrm{msec}$; tonic spikes $=33.8 \pm 1.1$ msec; $p<0.01$ ). The magnitude (integral) of the excitatory phase was also less for burst spikes compared with tonic spikes (Fig. 
$\left.4 B_{1}\right)$ (burst spikes $=0.024 \pm 0.001$; tonic spikes $=0.030 \pm 0.001$; $p<0.00001)$. This difference is not attributable to a decrease in the excitatory phase maximum (Fig. $4 C_{1}$ ) (burst spikes $=0.95 \pm$ 0.02 ; tonic spikes $=0.95 \pm 0.02 ; p<0.8)$ but rather a decrease in the duration of the excitatory phase (Fig. $4 D_{1}$ ) (burst spikes $=$ $32.7 \pm 0.5 \mathrm{msec}$; tonic spikes $=52.3 \pm 1.1 \mathrm{msec} ; p<0.00001$ ).

The suppressive phase of the spike-triggered average was also significantly different for burst spikes compared with tonic spikes. Similar to excitatory phase comparisons, the latency between suppressive phase maximum and neuronal response was significantly shorter for burst spikes compared with tonic spikes (Fig. $5 A_{1}$ ) (burst spikes $=41.7 \pm 1.1 \mathrm{msec}$; tonic spikes $=59.0 \pm$ $1.6 \mathrm{msec} ; p<0.00001)$. Unlike excitatory phase comparisons, however, the suppressive phase magnitude was greater for burst spikes compared with tonic spikes (Fig. $5 B_{1}$ ) (burst spikes $=$ $0.056 \pm 0.003$; tonic spikes $=0.016 \pm 0.001$ spikes; $p<0.00001)$. This increase is attributable to an increase in both the suppressive phase maximum (Fig. $5 C_{1}$ ) (burst spikes $=0.97 \pm 0.02$; tonic spikes $=0.41 \pm 0.03 ; p<0.00001)$ and the suppressive phase duration (Fig. $5 D_{1}$ ) (burst spikes $=81.9 \pm 2.4 \mathrm{msec}$; tonic spikes $=70.5 \pm 2.4 \mathrm{msec} ; p<0.01)$. These results are consistent with the view that bursts are triggered from a more hyperpolarized state than tonic spikes (see Discussion).

To address the possibility that any differences between the spike-triggered average of burst and tonic spikes are simply attributable to the long ISI $(>50 \mathrm{msec})$ that precedes the cardinal spike in a burst, we calculated spike-triggered averages for longISI tonic spikes and compared results to those of burst spikes (Figs. $4 A_{2}-D_{2}, 5 A_{2}-D_{2}$ ). With one exception, the magnitude of the excitatory phase, all of the differences in measures observed for burst versus tonic spikes were also present and statistically significant for burst versus long-ISI tonic spikes. This indicates that differences between burst and tonic responses are not solely a consequence of the long ISI that precedes bursts.

\section{Reliability of visually evoked bursts}

To examine the reliability of visually evoked bursts, we recorded responses from 26 LGN neurons during the presentation of a repeating, 5 sec clip of the contrast-reversing, sine-wave stimulus (see Materials and Methods). Responses, burst and tonic, of a typical LGN neuron are shown in Figure $6 \mathrm{~A}$. Burst probability, as a function of time, is shown in Figure $6 \mathrm{~B}$. A qualitative assessment of the response of this neuron to the repeating stimulus indicates that visually driven bursts can be both highly reliable and temporally precise.

To quantify the reliability and temporal precision of bursts, we calculated a reliability index using window sizes ranging from \pm 1 to $20 \mathrm{msec}$ (see Materials and Methods). On average, when a burst occurs at a given time during one repeat of the stimulus, there is approximately a $50 \%$ chance that a burst will occur at the same time ( $\pm 4 \mathrm{msec}$ ) on subsequent repeats of the stimulus (Fig. $6 C$, black curve). To determine whether the measured reliability of burst spikes is a property associated with the long ISI $(>50$ $\mathrm{msec}$ ) preceding each burst, we compared reliability index values for burst spikes and long-ISI tonic spikes. As shown in Figure 6C, burst spikes are more reliable than long-ISI tonic spikes over a range of temporal windows (up to $20 \mathrm{msec}$ ). Finally, we quantified the temporal precision of bursts by calculating the SD of the distribution of burst times (i.e., the peaks in Fig. $6 \mathrm{~B}$ ) for each of the neurons in our sample. Across multiple repeats of the same visual stimulus, results show that most bursts occur within a temporal window of $\pm 2-4$ msec (Fig. $6 D$ ).

Although burst spikes are significantly more reliable than
A

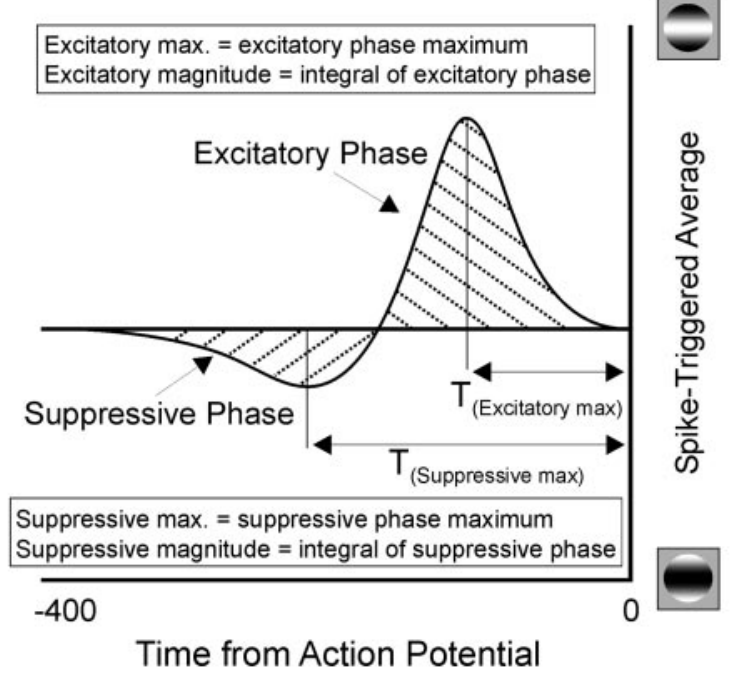

B

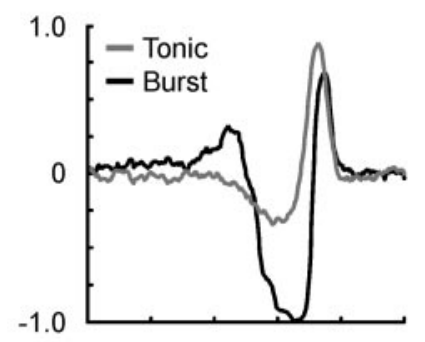

C
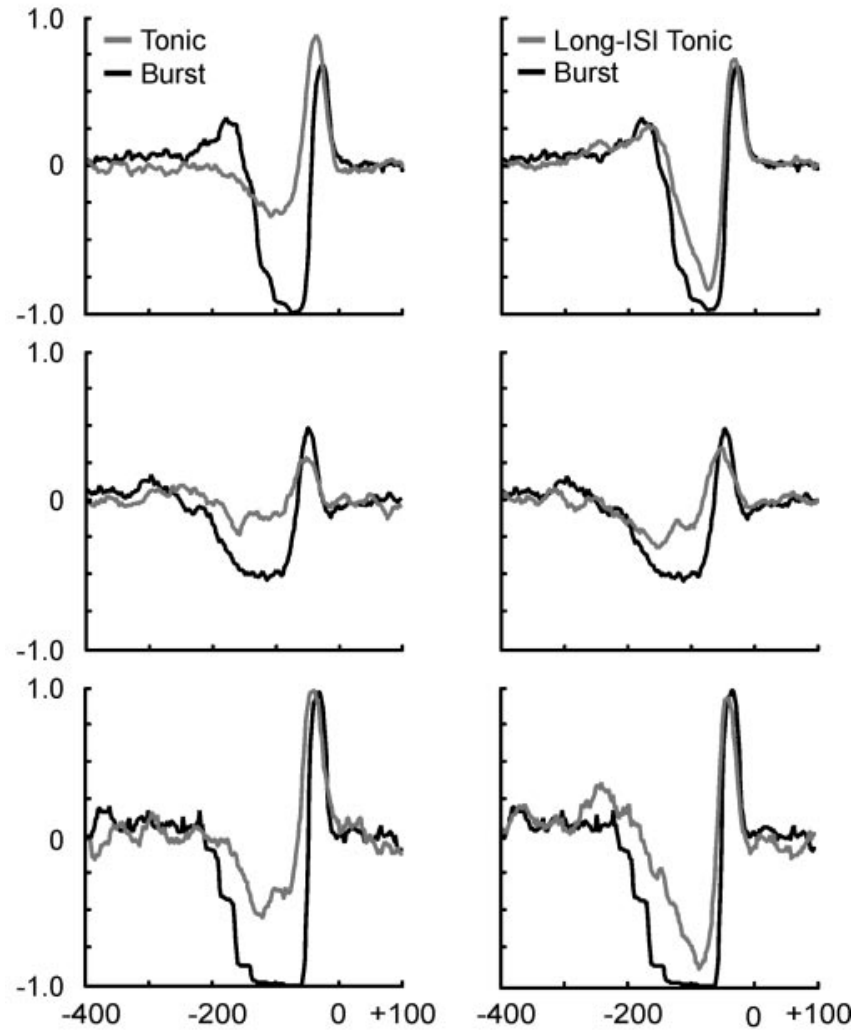

Time from Action Potential

(msec)

Figure 3. Spike-triggered averages. $A$, Features of the spike-triggered average were used to quantify temporal properties of the average stimulus to evoke burst, tonic, and long-ISI tonic spikes. B, Spike-triggered averages for burst spikes and tonic spikes from three representative LGN neurons. C, Spike-triggered averages for burst spikes and long-ISI tonic spikes from the same three representative neurons. Only bursts with ISIs that match those of long-ISI tonic spikes were included in this analysis.

long-ISI tonic spikes, it is important to note that burst reliability varied considerably across our sample of neurons. Specifically, burst reliability ranged from 0.01 to 0.9 (Fig. $7 A)($ mean $=0.45 \pm$ 0.06 , using a $5 \mathrm{msec}$ window). To determine whether burst reli- 


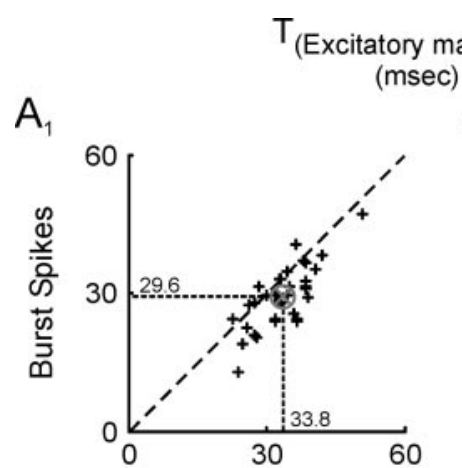

$A_{2}$

\section{Excitatory Magnitude}

(stimulus units)

$\mathrm{B}_{1}$

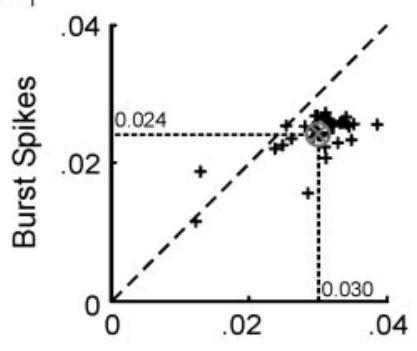

$\mathrm{B}_{2}$

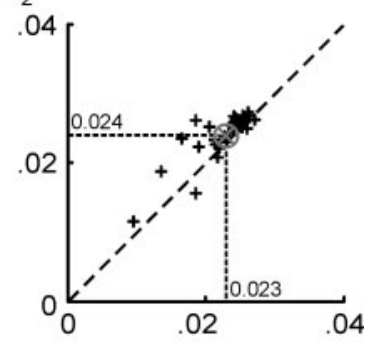

Excitatory Maximum

(stimulus units)

$\mathrm{C}_{1}$

$\mathrm{C}_{2}$
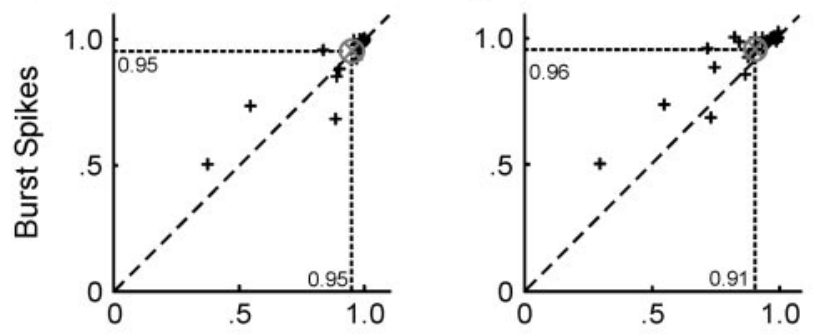

\section{Excitatory Duration}

(msec)

$\mathrm{D}_{1}$

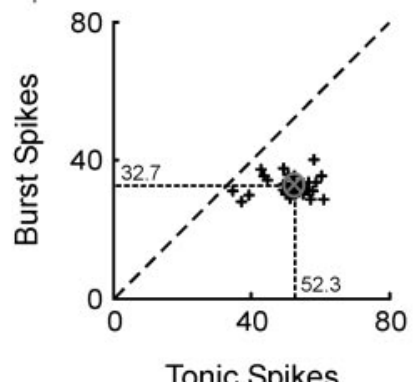

$\mathrm{D}_{2}$

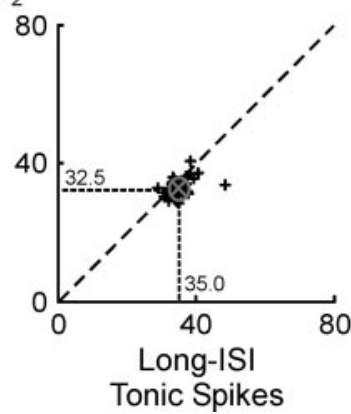

Figure 4. The excitatory phase of the spike-triggered average differs significantly for burst spikes compared with tonic spikes and long-ISI tonic spikes. $A_{1}, A_{2}$, The latency from excitatory phase maximum to neuronal response was significantly less for burst spikes compared with tonic spikes $\left(A_{1}\right)$ but not less for burst spikes compared with long-ISI tonic spikes $\left(A_{2}\right) . B_{1}, B_{2}$, The magnitude of the excitatory phase was significantly less for burst spikes compared with tonic spikes $\left(B_{1}\right)$ but not less for burst spikes compared with long-ISI tonic spikes $\left(B_{2}\right)$. The difference in excitatory phase magnitude did not reflect differences in the excitatory phase maximum of burst spikes compared with tonic spikes $\left(C_{1}\right)$ or long-ISI tonic spikes $\left(C_{2}\right)$ but did reflect differences in the duration of burst spikes compared with tonic spikes $\left(D_{1}\right)$ and long-ISI tonic spikes $\left(D_{2}\right)$.
$\mathrm{T}_{\text {(Suppressive maximum) }}$ (msec)

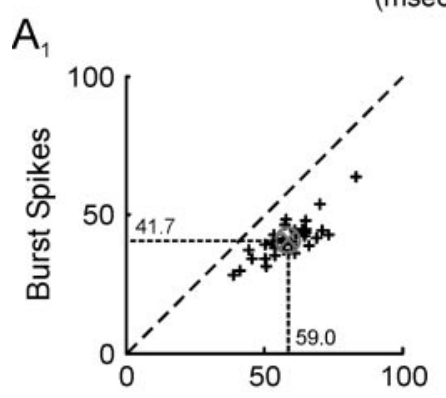

$\mathrm{A}_{2}$

\section{Suppressive Magnitude}

(stimulus units)

$\mathrm{B}_{1}$

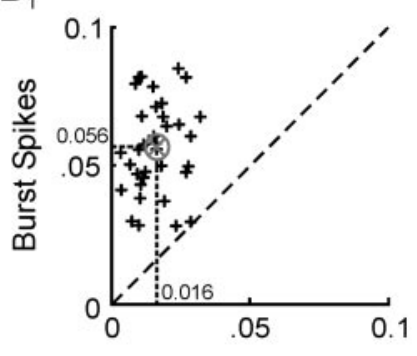

$\mathrm{B}_{2}$

\section{Suppressive Maximum \\ (stimulus units)}

$\mathrm{C}_{1}$

$\mathrm{C}_{2}$
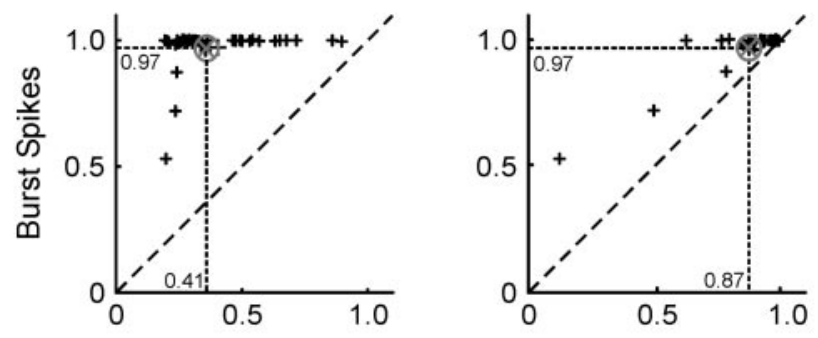

Suppressive Duration

(msec)

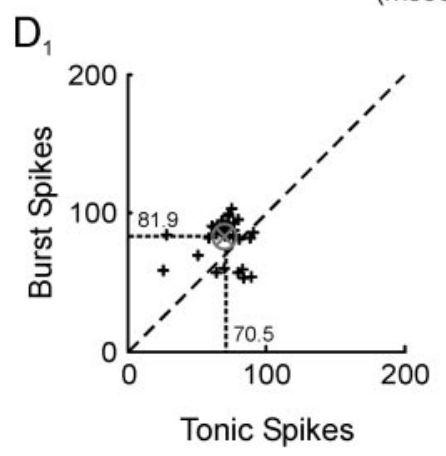

$\mathrm{D}_{2}$

Figure 5. The suppressive phase of the spike-triggered average differs significantly for burst spikes compared with tonic spikes and long-ISI tonic spikes. $A_{1}, A_{2}$, The latency from suppressive phase maximum to neuronal response was significantly less for burst spikes compared with tonic spikes $\left(A_{1}\right)$ and long-ISI tonic spikes $\left(A_{2}\right) . B_{1}, B_{2}$, The magnitude of the suppressive phase was significantly greater for burst spikes compared with tonic spikes $\left(B_{7}\right)$ and long-ISI tonic spikes $\left(B_{2}\right)$. The difference in suppressive phase magnitude of burst spikes reflects increases in the suppressive phase maximum of burst spikes compared with tonic spikes $\left(C_{7}\right)$ and long-ISI tonic spikes $\left(C_{2}\right)$ and increases in the duration of burst spikes compared with tonic spikes $\left(D_{1}\right)$ and long-ISI tonic spikes $\left(D_{2}\right)$. 
ability was related to burst frequency, we divided the LGN neurons in our sample into two groups (Fig. 7A), cells with low burst reliability $(<0.4 ; n=12)$ and cells with high burst reliability $(>0.4 ; n=14)$, and compared burst frequency. On average, cells with high burst reliability have a higher burst frequency than cells with low burst reliability (Fig. $7 B)(4.40 \pm 0.55$ vs $0.73 \pm 0.22$ bursts/sec, respectively). Although the two categories of cells are significantly different from each other, it is worth noting that both categories include cells with low burst frequency.

\section{Discussion}

This study addresses two fundamental questions about visually driven bursts in the LGN: (1) are bursts driven by distinct spatiotemporal patterns of visual stimulation, and (2) are visually driven bursts reliable? Using reverse-correlation analysis, our results show that the average stimulus to precede burst spikes is similar to, but significantly different from, the average stimulus to precede tonic spikes. Compared with tonic spikes, burst spikes occur with a shorter latency between stimulus and response and generally follow stimuli that provide increased drive to the receptive field center and even greater increased drive to the receptive field surround. Bursts also display a greater dependence on the transition from a suppressive stimulus to the preferred stimulus. Finally, results show that visually driven bursts can be highly reliable and temporally precise. In the sections below, we consider the possible mechanisms that underlie visually driven bursts and discuss the implications of our results for visual processing.
A

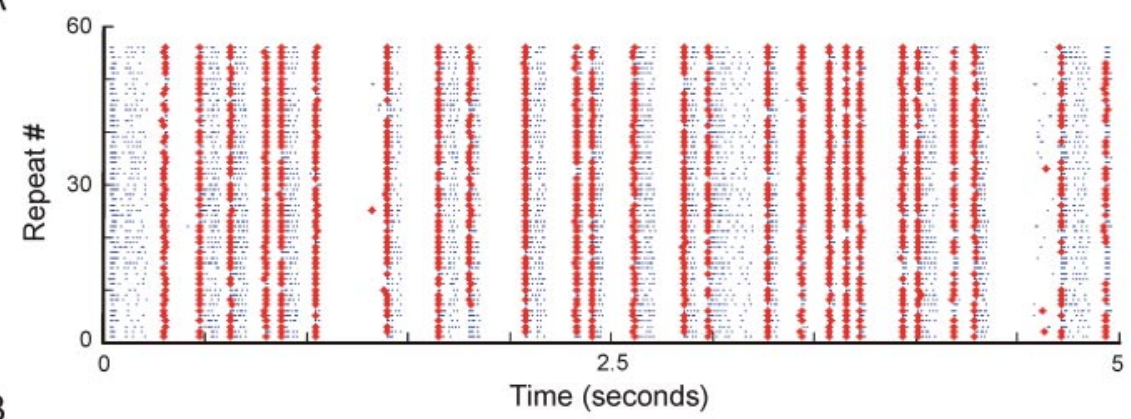

B

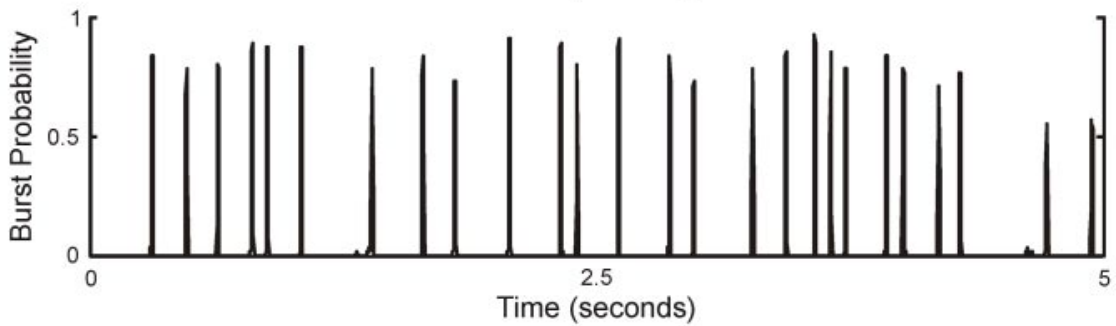

C
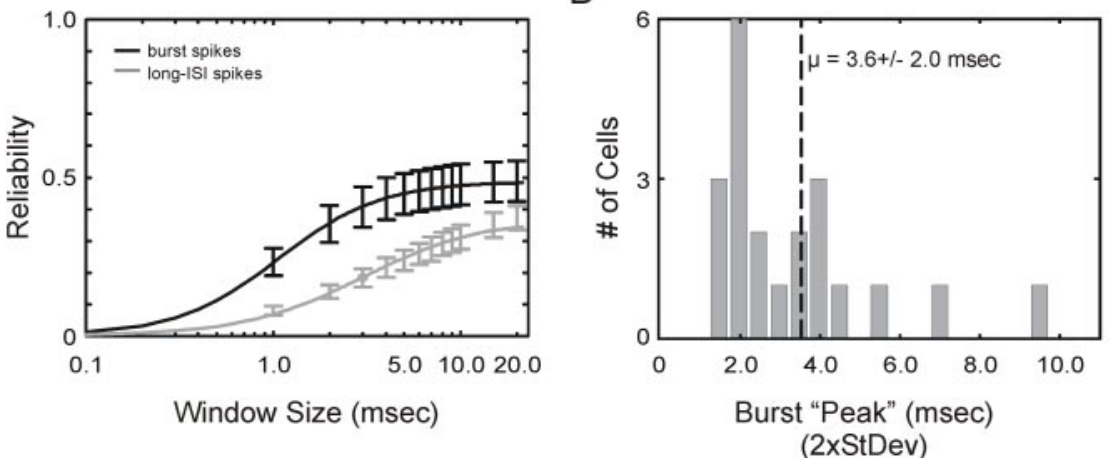

Figure 6. Burst spikes are reliable and temporally precise across multiple repeats of the same visual stimulus. $A$, Responses of a representative $L G N$ neuron (reliability index $=0.77$ ) presented with multiple repeats of the same 5 sec clip of the m-sequence modulated, contrast-reversing, sine-wave stimulus. Each line in the raster represents a different presentation of the visual stimulus. Cardinal spikes of a burst are indicated in red, and tonic spikes are indicated in blue. $B$, Burst probability as a function of time for the 5 sec period shown in A. Burst probability was calculated using the cardinal spike of each burst and a sliding $10 \mathrm{msec}$ window. C, Reliability (see Materials and Methods) is greater for burst spikes (black curve) compared with long-ISI tonic spikes (gray curve) over a range of temporal windows (1-20 msec). Error bars represent SEM. D, Cardinal spikes of bursts are temporally precise, as indicated from measures of the SD of burst peaks in burst probability plots (i.e., the peaks shown in B). Only peaks with $>25 \%$ reliability were used for this analysis. As a result, 21 of 26 cells are shown. The dashed line indicates the population mean.

\section{Visual stimuli and LGN bursts}

Several studies have examined and characterized the cellular mechanisms that underlie burst and tonic spikes in the thalamus. Most of these studies focus on the T-type $\mathrm{Ca}^{2+}$ channel and the low-threshold current these channels provide (Jahnsen and Llinás, 1984a,b; Zhou et al., 1997; Destexhe et al., 1998). Interestingly, the ability of these channels to generate low-threshold spikes depends critically on the membrane potential history of the neuron. When neurons are relatively depolarized, this channel is inactivated and depolarizing currents lead to tonic $\mathrm{Na}^{+}$ spikes. Under more hyperpolarized conditions, the channel becomes deinactivated, and depolarizing currents evoke a lowthreshold $\mathrm{Ca}^{2+}$ current that triggers a burst of $\mathrm{Na}^{+}$spikes. Because T-type $\mathrm{Ca}^{2+}$ channels need to be hyperpolarized for $>50$ msec to become deinactivated, this criterion has been applied with success to identifying low-threshold bursts with extracellular electrodes in vivo (Lo et al., 1991; Lu et al., 1992). Although we cannot be certain that $\mathrm{T}$-type $\mathrm{Ca}^{2+}$ channels are involved in the production of bursts measured in the current study, several properties of the visual stimulus that evoke bursts are consistent with their involvement. In particular, the increased dependence of bursts for a suppressive stimulus before the excitatory stimulus is consistent with the notion that suppressive stimuli can hyperpolarize LGN neurons (Singer et al., 1972; Martinez et al., 2003) and thereby contribute to deinactivating T-type $\mathrm{Ca}^{2+}$ channels. Along these lines, consistent with previous reports, we found that the suppressive phase of the spike-triggered average was significantly greater in both magnitude and duration for burst spikes compared with tonic spikes (Reinagel et al., 1999; Kepecs and Lisman, 2003).

In vitro studies report that deinactivation of $\mathrm{T}$-type $\mathrm{Ca}^{2+}$ channels can make an otherwise subthreshold current injection capable of evoking a burst (Lo et al., 1991; Sherman 1993). Based on this finding, one would expect bursts to require less excitatory drive from a visual stimulus than tonic spikes and certainly not more excitatory drive, as we report. Although we cannot be certain why burst spikes display an increased dependence on excitatory drive, it is tempting to speculate that the increased dependence reflects a dynamic relationship between low- 
A

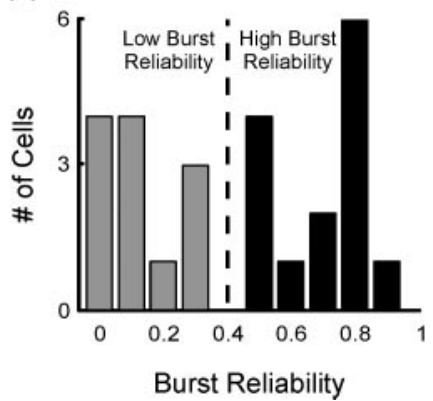

B

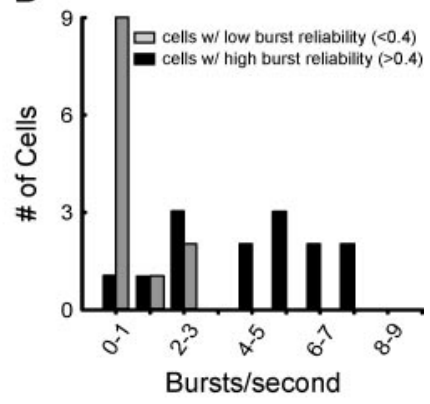

Figure 7. Relationship between burst reliability and burst rate. $A$, Histogram showing the distribution of burst reliability values for 26 neurons. Neurons with reliability values above and below 0.4 are arbitrarily divided into high and low burst categories, respectively. $B$, Burst reliability is correlated with burst rate; however, a small population of LGN neurons with low burst rate have high burst reliability.

threshold currents in the LGN and retinal drive during visual stimulation. Presumably, suppressive stimuli that hyperpolarize LGN neurons also hyperpolarize the retinal ganglion cells that provide their input. If retinal ganglion cells do not hyperpolarize to the same extent as LGN neurons, then it may be possible for the visual stimulus to deinactivate T-type $\mathrm{Ca}^{2+}$ channels in the LGN but not T-type $\mathrm{Ca}^{2+}$ channels in the retina. As a result, retinal ganglion cells would not have access to the low-threshold current and would require a stronger visual stimulus to reach threshold. In other words, a visual stimulus that decreases threshold in the LGN would also decrease drive from the retina. A stronger visual stimulus would then be needed to drive the retina to a level sufficient for the LGN to reach threshold. Although this line of reasoning is purely speculative, partial support for it comes from the findings that (1) retinal ganglion cells produce very few bursts, and (2) the average stimulus to evoke a response from a retinal ganglion cell has an increased surround to center ratio for spikes with interspike intervals $>50 \mathrm{msec}$ compared with spikes with interspike intervals $<10 \mathrm{msec}$ (Rathbun et al., 2003).

In principle, extra excitation could be supplied by simply scaling the average stimulus that precedes tonic spikes. If this were the case, then stimuli that evoke burst and tonic spikes would have similar surround to center ratios. Instead, we show that the surround to center ratio is greater for stimuli that evoke burst spikes. This differential increase in surround to center stimulation can be explained simply as reflecting differences in the excitatory drive needed to reach threshold for burst and tonic spikes. By convolving a model LGN receptive field with the m-sequence stimulus, we found that the surround to center ratio is indeed increased by simply increasing the threshold for spike generation. Given that the average stimulus to precede burst spikes has a greater surround to center ratio than that which precedes tonic spikes, the result is consistent with the proposal that more excitatory drive is needed to evoke burst spikes compared with tonic spikes.

Additional evidence supporting the idea that burst spikes require greater excitatory drive from a visual stimulus than tonic spikes comes from an examination of the temporal features of burst and tonic responses to visual stimuli. Using noisemodulated contrast-reversing stimuli, we found a significant decrease in the response latency and response duration of burst spikes compared with tonic spikes. In the retina, LGN, and visual cortex, there is a well documented inverse relationship between

stimulus strength and response latency (Shapley and Victor, 1978, 1981; Dean and Tolhurst, 1986; Sclar, 1987; Saul and Humphrey, 1990; Benardete et al., 1992; Reid et al., 1992; Carandini and Heeger, 1994; Albrecht, 1995; Kremers et al., 1997). Although indirect, the finding that burst spikes have a decreased latency compared with tonic spikes is consistent with the idea that, on average, burst spikes follow a stronger visual stimulus than tonic spikes.

A recent study using sparse-noise stimuli to compare burst and tonic receptive fields reports a decrease in the receptive field center of burst spikes compared with tonic spikes (Rivadulla et al., 2003). In contrast, we did not detect any differences in the spatial extent of stimuli that evoke burst and tonic spikes. This discrepancy may reflect differences in the stimuli used in the two studies. In the current study, we used a white-noise stimulus that captures both the center and surround structure of the LGN receptive field. The previous study used a sparse-noise stimulus that is generally less effective at capturing receptive field surrounds. Given that a major difference between burst and tonic receptive fields measured in the current study is in the strength of the surround, certain discrepancies in the results of the two studies are unavoidable. Nevertheless, we believe both sets of results are compatible with the hypothesis that a more effective stimulus is required to evoke burst spikes compared with tonic spikes.

\section{Reliability of LGN bursts}

Visual stimuli that include an abrupt transition from a suppressive to optimal state are particularly effective at driving LGN bursts. This finding suggests that visually driven bursts may play an important role in conveying information about the occurrence of these events to the cortex. The ability of a burst to be informative, however, depends not only on the filtering properties of a burst, but also on the probability (or reliability) that specific sequences of stimuli will evoke a burst. Using a repeating segment of a noise-modulated contrast-reversing grating, we found that LGN neurons reliably generate bursts with high temporal precision. This result is in agreement with previous findings that bursts can be temporally precise and capable of representing a relatively large amount of information (Guido et al., 1995; Guido and Sherman, 1998; Reinagel et al., 1999; Denning et al., 2003).

In general, we found a positive relationship between burst frequency and burst reliability. This is an important point to consider when relating results from anesthetized and alert animals. Because alert animals produce far fewer bursts than anesthetized animals (Guido and Weyand, 1995; Ramcharan et al., 2000; Swadlow and Gusev, 2001; Weyand et al., 2001; Royal et al., 2003), it seems reasonable to suggest that bursts would be less reliable in alert animals. Although this is a definite possibility, it is worth noting that a subset of the neurons in our sample that produced relatively few bursts still produced these bursts in a highly reliable manner. This finding raises the possibility that visually driven bursts may be highly reliable among a subset of LGN neurons in alert animals.

\section{Bursts and thalamocortical processing}

Our results show that LGN bursts can be driven by specific patterns of visual stimulation and that bursts can be highly reliable. Provided there exists a cortical readout for bursts, bursts appear well suited for conveying sensory information to cortex. Most studies agree that thalamocortical synapses experience synaptic depression (Stratford et al., 1996; Gil et al., 1999; Chung et al., 2002). If so, then the long ISI preceding the cardinal spike of a 
burst should allow for recovery from depression and thereby increase thalamocortical burst efficacy. Even if thalamocortical synapses experience modest depression (Boudreau and Ferster, 2003), the rapid train of spikes within a burst should experience temporal summation (Usrey et al., 2000) and thereby lead to a similar increase in burst efficacy. Although a direct measure of the effectiveness of LGN bursts for driving responses in visual cortex has yet to be performed, an equivalent study has been performed in the somatosensory pathway of the rabbit (Swadlow and Gusev, 2001). Results from that study clearly demonstrate that burst spikes are more effective than tonic spikes at driving cortical responses. If LGN bursts are similarly more effective at driving cortical responses, then visually driven bursts would seem to have all of the necessary ingredients to represent a distinct mode for processing and conveying visual information to the cortex.

\section{References}

Albrecht DG (1995) Visual cortex neurons in monkey and cat: effect of contrast on the spatial and temporal phase transfer functions. Vis Neurosci 12:1191-1210.

Benardete EA, Kaplan E, Knight BW (1992) Contrast gain control in the primate retina: $\mathrm{P}$ cells are not $\mathrm{X}$-like, some $\mathrm{M}$ cells are. Vis Neurosci 8:483-486.

Bishop PO, Burke W, Davis R (1958) Synapse discharge by single fibre in mammalian visual system. Nature 128:728-730.

Boudreau DE, Ferster D (2003) Synaptic depression in thalamocortical synapses of the cat visual cortex. Soc Neurosci Abstr 29:484.11.

Carandini M, Heeger DJ (1994) Summation and division by neurons in primate visual cortex. Science 264:1333-1336.

Chance FS, Nelson SB, Abbott LF (1998) Synaptic depression and the temporal response characteristics of V1 cells. J Neurosci 18:4785-4799.

Chung S, Li X, Nelson SB (2002) Short-term depression at thalamocortical synapses contributes to rapid adaptation of cortical sensory responses in vivo. Neuron 34:437-446.

Citron MC, Emerson RC, Ide LS (1981) Spatial and temporal receptive-field analysis of the cat's geniculocortical pathway. Vision Res 21:385-396.

Cleland BG, Dubin MW, Levick WR (1971) Simultaneous recording of input and output of lateral geniculate neurones. Nat New Biol 231:191-192.

Dean AF, Tolhurst DJ (1986) Factors influencing the temporal phase of response to bar and grating stimuli for simple cells in the cat striate cortex. Exp Brain Res 62:143-151.

Denning KS, Reid R, Reinagel P (2003) LGN burst state is controlled by visual signals. Soc Neurosci Abstr 29:699.12.

Destexhe A, Neubig M, Ulrich D, Huguenard J (1998) Dendritic lowthreshold calcium currents in thalamic relay cells. J Neurosci 18:3574-3588.

Gil Z, Connors BW, Amitai Y (1999) Efficacy of thalamocortical and intracortical synaptic connections: quanta, innervation, and reliability. Neuron 23:385-397.

Guido W, Sherman SM (1998) Response latencies of cells in the cat's lateral geniculate nucleus are less variable during burst than tonic firing. Vis Neurosci 15:231-237.

Guido W, Weyand T (1995) Burst responses in thalamic relay cells of the awake behaving cat. J Neurophysiol 74:1782-1786.

Guido W, Lu SM, Sherman SM (1992) Relative contributions of burst and tonic responses to the receptive field properties of lateral geniculate neurons in the cat. J Neurophysiol 68:2199-2211.

Guido W, Lu SM, Vaughan JW, Godwin DW, Sherman SM (1995) Receiver operating characteristic (ROC) analysis of neurons in the cat's lateral geniculate nucleus during tonic and burst response mode. Vis Neurosci 12:723-741.

Hubel DH, Wiesel TN (1961) Integrative action in the cat's lateral geniculate body. J Physiol (Lond) 155:385-398.

Jahnsen H, Llinás R (1984a) Electrophysiological properties of guinea-pig thalamic neurones: an in vitro study. J Physiol (Lond) 349:205-226.

Jahnsen H, Llinás R (1984b) Ionic basis for the electroresponsiveness and oscillatory properties of guinea-pig thalamic neurones in vitro. J Physiol (Lond) 349:227-247.
Jones JP, Palmer LA (1987) The two-dimensional spatial structure of simple receptive fields in cat striate cortex. J Neurophysiol 58:1187-1211.

Kepecs A, Lisman J (2003) Information encoding and computation with spikes and bursts. Network 14:103-118.

Kremers J, Weiss S, Zrenner E (1997) Temporal properties of marmoset lateral geniculate cells. Vision Res 37:2649-2660.

Lo FS, Lu SM, Sherman SM (1991) Intracellular and extracellular in vivo recordings of different response modes for relay cells of the cat's lateral geniculate nucleus. Exp Brain Res 83:317-328.

Lu SM, Guido W, Sherman SM (1992) Effects of membrane voltage on receptive field properties of lateral geniculate neurons in the cat: contributions of the low-threshold $\mathrm{Ca}^{2+}$ conductance. J Neurophysiol 68:1285-1298.

Martinez LM, Alonso JM, Hirsch JA (2003) Synaptic structure of receptive fields in the cat's early visual pathway. Soc Neurosci Abstr 29:910.19.

Mastronarde DN (1987) Two classes of single-input X-cells in cat lateral geniculate nucleus. II. Retinal inputs and the generation of receptive-field properties. J Neurophysiol 57:381-413.

McCormick DA, Bal T (1994) Sensory gating mechanisms of the thalamus. Curr Opin Neurobiol 4:550-556.

McCormick DA, Feeser HR (1990) Functional implications of burst firing and single spike activity in lateral geniculate relay neurons. Neuroscience 39:103-113.

Ramcharan EJ, Gnadt JW, Sherman SM (2000) Burst and tonic firing in thalamic cells of unanesthetized, behaving monkeys. Vis Neurosci 17:55-62.

Rathbun DL, Alitto HJ, Weyand TG, Usrey WM (2003) Spike-timing dependent receptive fields of cat retinal ganglion cells. Soc Neurosci Abstr 29:698.18.

Reid RC, Shapley RM (1992) Spatial structure of cone inputs to receptive fields in primate lateral geniculate nucleus. Nature 356:716-718.

Reid RC, Victor JD, Shapley RM (1992) Broadband temporal stimuli decrease the integration time of neurons in cat striate cortex. Vis Neurosci 9:39-45.

Reid RC, Victor JD, Shapley RM (1997) The use of m-sequences in the analysis of visual neurons: linear receptive field properties. Vis Neurosci 16:1015-1027.

Reinagel P, Godwin D, Sherman SM, Koch C (1999) Encoding of visual information by LGN bursts. J Neurophysiol 81:2558-2569.

Rivadulla C, Martinez L, Grieve KL, Cudeiro J (2003) Receptive field structure of burst and tonic firing in feline lateral geniculate nucleus. J Physiol (Lond) 553:601-610.

Royal DW, Sary G, Schall J, Casagrande V (2003) Are spike bursts and pseudo-bursts in the lateral geniculate nucleus (LGN) related to behavioral events? Soc Neurosci Abstr 29:699.16.

Saul AB, Humphrey AL (1990) Spatial and temporal response properties of lagged and nonlagged cells in cat lateral geniculate nucleus. J Neurophysiol 64:206-224.

Sclar G (1987) Expression of "retinal" contrast gain control by neurons of the cat's lateral geniculate nucleus. Exp Brain Res 66:589-596.

Shapley RM, Victor JD (1978) The effect of contrast on the transfer properties of cat retinal ganglion cells. J Physiol (Lond) 285:275-298.

Shapley RM, Victor JD (1981) How the contrast gain control modifies the frequency responses of cat retinal ganglion cells. J Physiol (Lond) 318:161-179.

Sherman SM (1993) Dynamic gating of retinal transmission to the visual cortex by the lateral geniculate nucleus. In: Thalamic networks for relay and modulation (Minciacchi D, Molinari M, Macchi G, Jones EG, eds), pp 61-79. Oxford: Pergamon.

Sherman SM (2001) A wake-up call from the thalamus. Nat Neurosci 4:344-346.

Singer W, Poppel E, Creutzfeldt O (1972) Inhibitory interaction in the cat's lateral geniculate nucleus. Exp Brain Res 14:210-226.

Steriade M (2001a) To burst, or rather, not to burst. Nat Neurosci 4:671.

Steriade M (2001b) Corticothalamic resonance, states of vigilance and mentation. Neuroscience 101:243-276.

Steriade M, Llinas RR (1988) The functional states of the thalamus and the associated neuronal interplay. Physiol Rev 68:649-742.

Steriade M, Jones EG, Llinas RR (1990) Thalamic oscillations and signaling. New York: Wiley. 
Stratford KJ, Tarczy-Hornoch K, Martin KA, Bannister NJ, Jack JJ (1996) Excitatory synaptic inputs to spiny stellate cells in cat visual cortex. Nature 382:258-261.

Sutter EE (1992) A deterministic approach to nonlinear systems analysis. In: Nonlinear vision: determination of neural receptive fields, function, and networks (Pinter R, Nabet B, eds), pp 171-220. Cleveland: CRC.

Swadlow HA, Gusev AG (2001) The impact of "bursting" thalamic impulses at a neocortical synapse. Nat Neurosci 4:402-408.

Usrey WM (2002) Spike timing and visual processing in the retinogeniculocortical pathway. Philos Trans R Soc Lond B Biol Sci 357:1729-1737.

Usrey WM, Reppas JB, Reid RC (1999) Specificity and strength of retinogeniculate connections. J Neurophysiol 82:3527-3540.

Usrey WM, Alonso JM, Reid RC (2000) Synaptic interactions between thalamic inputs to simple cells in cat visual cortex. J Neurosci 20:5461-5467.

Usrey WM, Sceniak MP, Chapman B (2003) Receptive fields and response properties of neurons in layer 4 of ferret visual cortex. J Neurophysiol 89:1003-1015.

Weyand TG, Boudreaux M, Guido W (2001) Burst and tonic response modes in thalamic neurons during sleep and wakefulness. J Neurophysiol 85:1107-1118.

Wolfe J, Palmer LA (1998) Temporal diversity in the lateral geniculate nucleus of cat. Vis Neurosci 15:653-675.

Zhou Q, Godwin DW, O’Malley DM, Adams PR (1997) Visualization of calcium influx through channels that shape the burst and tonic firing modes of thalamic relay cells. J Neurophysiol 77:2816-2825. 